\title{
COMPACT AND WEAKLY COMPACT COMPOSITION OPERATORS ON SPACES OF VECTOR VALUED CONTINUOUS FUNCTIONS
}

\author{
R. K. SINGH AND W. H. SUMMERS
}

\begin{abstract}
We characterize the compact and weakly compact weighted composition operators on the Banach space $C_{p}(T, X)$ of those continuous functions from a completely regular Hausdorff space $T$ into a Banach space $X$ which have relatively compact range.
\end{abstract}

The compact weighted endomorphisms of the Banach algebra $C(T)$ of all real or complex valued continuous functions on a compact Hausdorff space $T$ have been characterized by $\mathrm{H}$. Kamowitz [1]. In particular, given $u \in C(T)$ and a continuous function $\phi: T \rightarrow T$, Kamowitz has shown that the weighted composition operator $u C_{\phi}: C(T) \rightarrow C(T)$ (defined by $u C_{\phi}(f)(t)=u(t) f(\phi(t))$ for $f \in C(T)$ and $t \in T$ ) is compact if and only if each component of $N(u)=\{t \in T: u(t) \neq 0\}$ is contained in some open set $V$ such that $\phi \mid V$ is a constant function.

For an arbitrary completely regular Hausdorff space $T$, however, if $C(T)$ is replaced by the supremum normed algebra $C_{b}(T)$ of all scalar valued bounded continuous functions on $T$, Kamowitz's condition does not guarantee that a weighted composition operator on $C_{b}(T)$ is compact. Indeed, this can be readily seen through an obvious modification of the example constructed by Kamowitz [1, p. 519] to show that $u C_{\phi}$ can fail to be compact even when $\phi$ is constant on each component of $N(u)$. A closer look at this same example also suggests a possible way around the problem. In case $T$ is compact, Kamowitz's result immediately yields that the following condition is at least necessary in order for $u C_{\phi}$ to be compact:

$$
\phi(\{t \in T:|u(t)| \geq \varepsilon\}) \text { is finite for each } \varepsilon>0 ;
$$

the key to Kamowitz's example lies in the fact that this direct link between $\phi$ and $u$ does not hold.

We will show that $(*)$ is both necessary and sufficient for a weighted composition operator on $C_{b}(T)$ to be compact, whereby $(*)$ is equivalent to Kamowitz's condition in case $T$ is compact. More importantly, we will demonstrate that $(*)$ serves as well to characterize the weakly compact weighted composition operators on $C_{b}(T)$. Since our methods allow, we shall actually place our results in the broader context of weighted composition operators on spaces of vector valued continuous functions.

Preliminaries. In what follows, we will use $X$ to denote a (nontrivial) real or complex Banach space, while $S$ and $T$ will denote completely regular Hausdorff spaces. The set of all continuous functions from $S$ into $T$ will be designated by

Received by the editors February 26, 1986.

1980 Mathematics Subject Classification (1985 Revision). Primary 47B05, 47B38. 
$C(S, T)$, and we will let $C_{p}(T, X)$ denote the vector space consisting of all $f \in$ $C(T, X)$ for which $f(T)$ is relatively compact in $X$. Further, we shall hereafter assume that $C_{p}(T, X)$ is supplied with the topology of uniform convergence on $T$ and denote the corresponding norm by $\|\cdot\|_{\infty}$. In case $T$ happens to be cornpact, we will simply write $C(T, X)$ instead of $C_{p}(T, X)$. Also, we shall use $C_{b}(T)$ in place of $C_{p}(T, X)$ when $X \in\{\mathbf{R}, \mathbf{C}\}$.

Each $\phi \in C(S, T)$ determines a continuous linear operator $C_{\phi}: C_{p}(T, X) \rightarrow$ $C_{p}(S, X)$ defined by setting $C_{\phi} f=f \circ \phi$ for $f \in C_{p}(T, X)$, and we will refer to $C_{\phi}$ as the composition operator induced by $\phi$. Clearly, if two functions $\phi, \psi \in C(S, T)$ induce the same composition operator, then $\phi=\psi$. For $u \in C_{b}(T)$ and $\phi \in C(T, T)$, the corresponding weighted composition operator $f \rightarrow u(f \circ \phi)$ on $C_{p}(T, X)$ will be denoted by $u C_{\phi}$. Finally, for $F \subseteq T$, we shall let $R_{F}$ denote the restriction operator from $C_{p}(T, X)$ into $C_{p}(F, X)$; i.e., $R_{F} f=f \mid F$ for each $f \in C_{p}(T, X)$.

1. Compact and weakly compact composition operators. We begin by observing that the study of compactness for composition operators can be reduced to the case where the underlying topological spaces are compact. As usual, the Stone-Cech compactification of a completely regular Hausdorff space $T$ is denoted by $\beta T$.

1.1. LEMMA. Let $\phi \in C(S, T)$, where $S$ and $T$ are completely regular Hausdorff spaces, let $\hat{\phi}: \beta S \rightarrow \beta T$ denote the Stone extension of $\phi$, and assume that $X$ is a Banach space. Then

$$
C_{\hat{\phi}}: C(\beta T, X) \rightarrow C(\beta S, X)
$$

is a compact (respectively, weakly compact) operator if and only if $C_{\phi}$ is a compact (respectively, weakly compact) operator.

Proof. For $f \in C_{p}(T, X)$, let $\beta(f)=\hat{f}$ be the Stone extension of $f$ to $\beta T$. Then $\beta: C_{p}(T, X) \rightarrow C(\beta T, X)$ is a surjective isometry, and the same is true for $R_{S}: C(\beta S, X) \rightarrow C_{p}(S, X)$. Moreover, given $f \in C_{p}(T, X)$,

$$
R_{S}\left(C_{\hat{\phi}} \hat{f}\right)=R_{S}(\hat{f} \circ \hat{\phi})=\hat{f} \circ \phi=C_{\phi} f
$$

from which the desired conclusion is now immediate.

1.2. THEOREM. Let $S$ and $T$ be completely regular Hausdorff spaces, fix $\phi \in$ $C(S, T)$, and assume that $X$ is a Banach space. Then

(1) $C_{\phi}: C_{p}(T, X) \rightarrow C_{p}(S, X)$ is a compact operator if and only if (i) $X$ is finite dimensional and (ii) $\phi(S)$ is finite;

(2) $C_{\phi}$ is a weakly compact operator if and only if (i) $X$ is reflexive and (ii) $\phi(S)$ is finite.

Proof. Since $\phi(S)$ is finite exactly when $K=\hat{\phi}(\beta S)$ is finite, it will suffice to show that $C_{\phi}$ is a compact (respectively, weakly compact) operator if and only if $C(K, X)$ is finite dimensional (respectively, reflexive). To this end, we first note that $W=R_{K}(C(\beta T, X))$ is a vector subspace of $C(K, X)$ which, additionally, is a module over the algebra $C(K)$. By the Stone-Weierstrass theorem for modules (cf. [2, p. 1023]), we therefore see that $W$ is dense in $C(K, X)$. Moreover, setting $G=\left\{f \in C(K, X):\|f\|_{\infty}<1\right\}$, if $f \in W \cap G$, then there clearly exists $g \in B=$ $\left\{h \in C(\beta T, X):\|h\|_{\infty} \leq 1\right\}$ such that $R_{K} g=f$. Combining these latter two facts, it follows that $G \subseteq \operatorname{cl}(G \cap W) \subseteq \operatorname{cl}\left(R_{K}(B)\right)$ whence $\operatorname{cl}(G)=\operatorname{cl}\left(R_{K}(B)\right)$. Next, 
let us define $\Phi: C(K, X) \rightarrow C(\beta S, X)$ by putting $\Phi f=f \circ \hat{\phi}$ for $f \in C(K, X)$. Since $\Phi$ is a linear isometry and $\Phi \circ R_{K}=C_{\hat{\phi}}$, we conclude that $\operatorname{cl}(G)$ is compact (respectively, weakly compact) if and only if $C_{\hat{\phi}}$ is a compact (respectively, weakly compact) operator. In view of the preceding lemma, this serves to complete the argument.

REMARK. As Theorem 1.2 makes plain, the classes of compact and weakly compact composition operators from $C_{p}(T, X)$ into $C_{p}(S, X)$ fail to coincide precisely when the Banach space $X$ is infinite dimensional and reflexive. Of course, both classes are trivial in case $X$ is not reflexive.

2. The weighted case. We are now in a position to establish the promised extension of Kamowitz's result on compact weighted endomorphisms. As a matter of notational convenience in the sequel, if $u \in C_{b}(T)$ and $\varepsilon>0$, we put $N(u, \varepsilon)=$ $\{t \in T:|u(t)| \geq \varepsilon\}$.

2.1. THEOREM. Let $T$ be a completely regular Hausdorff space, fix $\phi \in$ $C(T, T)$, take $u \in C_{b}(T)$ so that $u$ is not identically zero on $T$, and assume that $X$ is a Banach space. Then

(1) the weighted composition operator $u C_{\phi}$ on $C_{p}(T, X)$ is compact if and only if (i) $X$ is finite dimensional and (ii) $\phi(N(u, \varepsilon))$ is finite for every $\varepsilon>0$;

(2) $u C_{\phi}$ is a weakly compact operator if and only if (i) $X$ is reflexive and (ii) $\phi(N(u, \varepsilon))$ is finite for every $\varepsilon>0$.

PROOF. To begin, assume that $u C_{\phi}$ is a compact (respectively, weakly compact) operator on $C_{p}(T, X)$, and let $\varepsilon>0$; we may suppose that $N(u, \varepsilon) \neq \varnothing$. Now, setting $S=N(u, \varepsilon)$ and $\psi=\phi \mid S$, let us consider the composition operator $C_{\psi}: C_{p}(T, X) \rightarrow C_{p}(S, X)$ induced by $\psi$, and put $B=\left\{f \in C_{p}(T, X):\|f\|_{\infty} \leq 1\right\}$. Since

$$
C_{\psi}(B)=R_{S}\left(C_{\phi}(B)\right)=\frac{1}{u \mid S} R_{S}\left(u C_{\phi}(B)\right)
$$

$C_{\psi}$ is a compact (respectively, weakly compact) operator. According to Theorem 1.2, therefore, $X$ is finite dimensional (respectively, reflexive) and $\psi(S)=$ $\phi(N(u, \varepsilon))$ is finite.

Turning to the converse, let us assume that $X$ is finite dimensional and that $\phi(N(u, \varepsilon))$ is finite for each $\varepsilon>0$. Again setting $B=\left\{f \in C_{p}(T, X):\|f\|_{\infty} \leq 1\right\}$, we note in passing that $u C_{\phi}(B)(T)=\{u(t) f(\phi(t)): f \in B, t \in T\}$ is relatively compact in $X$ under these circumstances. Now, given $\varepsilon>0$, we choose $\eta \in(0, \varepsilon / 2)$ so that $S=N(u, \eta) \neq \varnothing$, and put $\psi=\phi \mid S$. By Theorem 1.2, we then also have that $C_{\psi}(B)$ is relatively compact in $C_{p}(S, X)$, whereby the same is true for $(u \mid S) C_{\psi}(B)$. Consequently, according to [3, Theorem 2.2], there exists a finite cover $\left(T_{i}\right)_{i=1}^{n}$ of $S$ such that $\|u(s) f(\phi(s))-u(t) f(\phi(t))\| \leq \varepsilon$ for all $f \in B$ whenever $s, t \in T_{i}$, $i=1, \ldots, n$. Putting $T_{n+1}=T \backslash S$, since $\left(T_{i}\right)_{i=1}^{n+1}$ covers $T$ and

$$
\|u(s) f(\phi(s))-u(t) f(\phi(t))\| \leq|u(s)|+|u(t)|<\varepsilon
$$

for all $f \in B$ when $s, t \in T_{n+1}$, another application of [3, Theorem 2.2] yields that $u C_{\phi}(B)$ is relatively compact in $C_{p}(T, X)$, which completes the argument for the case at hand. Finally, let us assume that $X$ is reflexive and that $\phi(N(u, \varepsilon))$ is finite for each $\varepsilon>0$. In order to show that $u C_{\phi}(B)$ is still at least weakly relatively 
compact in $C_{p}(T, X)$, we choose sequences $\left(f_{n}\right)$ in $B,\left(t_{m}\right)$ in $T$, and $\left(x_{m}^{\prime}\right)$ in $X^{\prime}$ with $\left\|x_{m}^{\prime}\right\| \leq 1$ for each $m \in \mathbf{N}$ such that

$$
\lim _{n} \lim _{m}\left\langle u\left(t_{m}\right) f_{n}\left(\phi\left(t_{m}\right)\right), x_{m}^{\prime}\right\rangle=\alpha
$$

and

$$
\lim _{m} \lim _{n}\left\langle u\left(t_{m}\right) f_{n}\left(\phi\left(t_{m}\right)\right), x_{m}^{\prime}\right\rangle=\beta
$$

for some $\alpha, \beta \in \mathbf{K}$, where $\mathbf{K}$ denotes the scalar field. Without loss of generality, we may suppose that there exists $\gamma \in \mathbf{K}$ such that $\lim _{m \rightarrow \infty} u\left(t_{m}\right)=\gamma$. This being the case, if $\gamma=0$, then $\alpha=\beta=0$. Otherwise, choosing $\varepsilon \in(0,|\gamma|)$, we may further suppose that $\left(t_{m}\right)$ is a sequence in $S=N(u, \varepsilon)$. From Theorem 1.2, however, if follows that $(u \mid S) C_{\phi \mid S}(B)$ is weakly relatively compact in $C_{p}(S, X)$, and the interchangeable double limits criterion for weak compactness as given in [4, Theorem 2.1] now yields that $\alpha=\beta$ in any event. A second application of $[4$, Theorem 2.1] consequently shows that $u C_{\phi}(B)$ is indeed weakly relatively compact in $C_{p}(T, X)$ as was required to complete the proof.

2.2. COROLLARY. Let $T$ be a completely regular Hausdorff space, fix $\phi \in$ $C(T, T)$, and take $u \in C_{b}(T)$. The following are equivalent in case the Banach space $X$ is finite dimensional:

(1) $u C_{\phi}$ is a compact operator on $C_{p}(T, X)$;

(2) $u C_{\phi}$ is a weakly compact operator on $C_{p}(T, X)$;

(3) $\phi(N(u, \varepsilon))$ is finite for every $\varepsilon>0$.

\section{REFERENCES}

1. H. Kamowitz, Compact weighted endomorphisms of $C(X)$, Proc. Amer. Math. Soc. 83 (1981), 517-521.

2. J. B. Prolla, Weighted approximation of continuous functions, Bull. Amer. Math. Soc. 77 (1971), 1021-1024.

3. W. M. Ruess and W. H. Summers, Compactness in spaces of vector valued continuous functions and asymptotic almost periodicity, Math. Nachr. (to appear).

4. _ Integration of asymptotically almost periodic functions and weak asymptotic almost periodicity (to appear).

Department of Mathematics, University of JAmmu, Jammu-18 00 01, INDia

Department of Mathematical Sciences, University of ARKansas, FayetteVILLE, ARKANSAS 72701 